\title{
SIGNIFICANCE OF INCORPORATING HETEROGENEITY IN A NON-CONTINUUM MACROSCOPIC MODEL FOR DENSITY ESTIMATION
}

\author{
Ajitha Thankappan ${ }^{1}$, Lelitha Vanajakshi ${ }^{1}$, Shankar C. Subramanian ${ }^{2}$ \\ ${ }^{1}$ Dept of Civil Engineering, Indian Institute of Technology Madras, India \\ ${ }^{2}$ Dept of Engineering Design, Indian Institute of Technology Madras, India
}

Submitted 3 November 2012; resubmitted 28 December 2012; accepted 15 February 2013

\begin{abstract}
The heterogeneity of traffic and the lack of lane discipline on the roads in India and other developing countries add complexity to the analysis and modeling of traffic. It is generally believed that it is important to take heterogeneity into account in traffic modeling. The aim of the present study is to check the validity of this assumption by analyzing the effect of incorporating heterogeneity in a macroscopic level traffic flow analysis. The application considered is real-time congestion analysis on Indian roads. Traffic density is considered as the congestion indicator. The measurement of density is difficult since it is a spatial parameter. It is usually estimated from other traffic parameters that can be readily measured using available sensors. A model-based estimation scheme using Kalman filtering has been employed to estimate traffic density. A non-continuum macroscopic model was attempted based on the lumped parameter approach. All the traffic variables were quantified without considering traffic lanes in order to take into account the lack of lane discipline. The effect of heterogeneity has been studied by incorporating static values of Passenger Car Units (PCU), dynamic values of Two Wheeler Units (TWU) and considering different classes of vehicles explicitly in the modeling process. The proposed estimation schemes without and with heterogeneity have been compared. The results have been corroborated using data collected from a road stretch in Chennai, India. The study shows that the significance of incorporating heterogeneity into the modeling of mixed traffic at the macroscopic level was not very significant.
\end{abstract}

Keywords: macroscopic modeling; non-continuum models; heterogeneous traffic; density estimation; extended Kalman filter.

Reference to this paper should be made as follows: Thankappan, A.; Vanajakshi, L.; Subramanian, S. C. 2014. Significance of incorporating heterogeneity in a non-continuum macroscopic model for density estimation, Transport 29(2): 125-136. http://dx.doi.org/10.3846/16484142.2014.928789

\section{Introduction}

Analysis and modeling of traffic flow is essential for understanding the traffic flow phenomena and for planning, design and management of transportation systems. The heterogeneous traffic conditions existing in India and other developing countries are highly complex and differ from the homogeneous and lane disciplined traffic seen in developed countries. Modeling of heterogeneous traffic is challenging due to the typical features that characterize a heterogeneous system such as the presence of several vehicle types and the absence of lane discipline. In general, it is assumed that to characterize such a system with reasonable accuracy, it is essential to incorporate heterogeneity into the modeling process.
To incorporate the effect of heterogeneity, the common approach is to use passenger car equivalents. However, there are no reported systematic studies analyzing the effect of this approach in the performance of traffic flow models.

There are many types of traffic flow models reported in literature, including microscopic and macroscopic models. Among these, microscopic models are complex and computationally intensive and hence not usually preferable for real time applications. Macroscopic models are more appropriate for applications where computational effort is crucial. Most of the available macroscopic traffic flow models treat traffic as a continuum (Hoogendoorn, Bovy 2001). However, this approximation is not accurate since the number of vehicles in a

Corresponding author: Lelitha Vanajakshi

E-mail: lelitha@iitm.ac.in 
road stretch is not sufficient enough to consider it as a continuum (Tyagi et al. 2009). Furthermore, the available macroscopic models may not be able to take into account some of the specific characteristics of Indian traffic such as absence of lane discipline and heterogeneity of traffic.

The studies reported on modeling of heterogeneous traffic are limited. The multi-class concept of incorporating heterogeneity has been used in a few traffic flow modeling studies by extending the continuum models developed for homogeneous traffic (Wong, Wong 2002; Logghe, Immers 2003; Tang et al. 2009). In India, apart from a few preliminary studies on macroscopic modeling (Padiath et al. 2009, 2010; Anand et al. 2011), there are no comprehensive studies in this respect. None of them analyzed the effect of incorporating heterogeneity of traffic in characterizing Indian traffic and evaluated its performance for real-time applications.

This study presents a non-continuum based lumped parameter macroscopic traffic flow model for the estimation of traffic density (the number of vehicles occupying a given length of roadway) under heterogeneous traffic conditions. Mathematical models of dynamic systems are typically used for estimation and control applications. The scope of the present study is to develop a density estimation scheme using a mathematical model for the traffic flow. The aspect of control of the present traffic system (a relevant study can be seen in Péter (2012)) investigated as part of a future study.

A model-based approach using techniques such as the Kalman filter is appropriate for traffic flow modeling applications that require estimation of traffic density, due to its ability to account for the uncertainty associated with the traffic flow phenomena. This is particularly relevant to heterogeneous traffic conditions where the randomness associated with traffic is high. Another advantage of this approach is that, it is recursive in nature, so that, unlike other historic methods, there is no need for a historic database. This is advantageous in places like India, where a database is not available due to absence of traffic sensors.

The base equation of the model proposed in this study has been formulated based on the conservation of vehicles inside a section of roadway and the auxiliary equation has been formulated using steady-state speeddensity relationships developed for the traffic under study. The two important spatial parameters - density and aggregate space-mean speed - that characterize traffic flow have been considered as the variables to be estimated in this model.

In order to account for the lack of lane discipline, all traffic variables have been quantified without considering traffic lanes. Thus, the model was first developed without considering heterogeneity and then heterogeneity was incorporated at an aggregate level by converting the heterogeneous traffic into a homogeneous equivalent as well as by including explicitly the different classes of vehicles into the model. In order to aggregate the heterogeneous traffic into a homogeneous equivalent, both static values of Passenger Car Units (PCU) and dynamic values of Two Wheeler Units (TWU) were used. Using these models, estimation schemes have been developed based on the Kalman filtering technique. The proposed schemes have been corroborated using data collected from sections along an urban arterial in Chennai and finally the significance of incorporating heterogeneity at a macroscopic level has been evaluated based on the performance of these estimation schemes.

\section{The Proposed Non-Continuum Model}

A non-continuum model has been proposed in this study for characterizing the system. The model was formulated based on the lumped parameter approach. Here, the physical system under study is divided into lumps or segments and within each segment, the aggregate characteristics such as velocity, pressure, density etc., may vary with time, but are assumed to be uniform over the segment. When this is applied to roadways, within a small section of roadway, the spatial variation of traffic variables (such as density, speed, etc.) is neglected and it is assumed that the variables depend only on time. A reasonable section length for this assumption to hold good is about $1-1.5 \mathrm{~km}$, the usual spacing between automated data collection sensors. The section length $L$ in this study has therefore been selected in this range. To apply this procedure to longer roadways, the section can be divided into sub-sections of lengths in this range. The lumped parameter approach results in the governing equations of the model being ordinary differential equations (in the continuous time domain) and ordinary difference equations (in the discrete time domain).

In order to formulate the model equations, a typical road segment between two data collection points, having a length $L$, is considered as shown in Fig. 1 .

The density and the average space mean speed of traffic, which are two spatial parameters difficult to measure from field, were considered as the macroscopic state variables in this study. The governing equation for density was formulated based on the conservation of vehicles inside the section as follows.

Let $N(k)$ denote the number of vehicles inside the section at the $k$ th instant of time. Then, the conservation of vehicles inside the section for a time step of $h$ can be described as:

$$
N(k+1)=N(k)+h\left(q_{\text {en }}(k)-q_{\text {ex }}(k)+q_{\text {side }}(k)\right),
$$

where: $q_{e n}(k)$ is the flow rate at which vehicles are entering into the section; $q_{e x}(k)$ is the flow rate at which vehicles are exiting from the section; $q_{\text {side }}(k)$ is the net flow rate at which the vehicles are entering into the section from the side road in the time interval $(k, k+1)$.

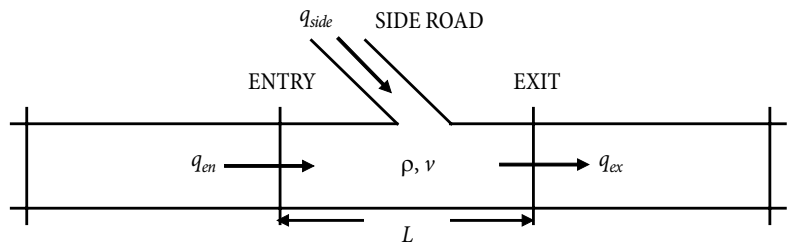

Fig. 1. Schematic diagram of a typical road section 
Dividing Equation (1) by the length of the section $L$ results in the governing equation for density as:

$$
\rho(k+1)=\rho(k)+h\left(q_{\text {en }}(k)-q_{\text {ex }}(k)+q_{\text {side }}(k)\right),
$$

where: $\rho(k+1)$ denotes the density inside the section at the $(k+1)$ th instant of time.

The above equation solely depends on the measured values of flow rates at which vehicles are entering into the section, exiting from the section and the net flow rate at which vehicles are entering into the section from side road. Thus, the accuracy of the methodology depends heavily on the accuracy of the measured flow values. If the sensors work perfectly, this method is appropriate. However, in reality, the sensors may not always work perfectly (Chen, May 1987; Turner et al. 2000; Vanajakshi, Rilett 2004). When there is a sensor malfunction, the flow data is affected more than other parameters such as speed and occupancy because flow data are reported as a cumulative number, whereas speed and occupancy data are averaged over the accumulation time interval. Hence, the effect of a sensor malfunction, such as missing vehicles, has less impact on speed and occupancy in comparison to flow data. This is a serious issue for Indian traffic conditions where none of the existing automated sensors can count all the parallel moving vehicles within the same lane. Average speed is a variable that can be obtained more accurately, especially under congested Indian traffic conditions. In the present study, the exit end of the section was assumed to experience more congestion due to the proximity to a signal downstream than other sections and hence prone to flow measurement errors. Hence, $q_{e x}$ in the above equation was written in terms of the average space-mean speed $v$ using the fundamental equation of traffic flow as:

$$
q_{\text {ex }}(k)=\rho(k) \cdot v(k) \text {. }
$$

Substituting Equation (3) in Equation (2) resulted in:

$$
\begin{aligned}
& \rho(k+1)=\rho(k)+\frac{h}{L} \times \\
& \left(q_{\text {en }}(k)-\rho(k) \cdot v(k)+q_{\text {side }}(k)\right),
\end{aligned}
$$

where: $\rho(k+1)$ denotes the density inside the section at the $(k+1)$ th instant of time. This substitution can be made for the end that is more congested, which in this case is the exit location.

The governing equation for average space mean speed of vehicles inside the section was formulated as a dynamic speed equation by incorporating a steady state speed-density relationship developed for the traffic being analyzed. The optimum relation was chosen based on the motive of minimizing the error $e$ between the speed values estimated using this steady state speeddensity relation $v(\rho)$ and the observed speed values $v$, that is, $e=v(\rho)-v$. The time evolution of this error was hypothesized to be governed by:

$$
\frac{d e}{d t}=-a \cdot e(t),
$$

where: the parameter $a$ is selected to be positive.
This equation is a linear homogeneous Ordinary Differential Equation (ODE) with a unique solution, $e(t)=e(0) \cdot \exp (-a \cdot t)$, (Coddington 1989), where $e(0)$ is the initial error (can be either positive or negative), which will converge to zero with time. Although there may be other choices for describing the time evolution of the error function, an exponentially decaying error function has been chosen in this study since its performance will be comparably good to any alternate choice. This approach has been applied in other studies involving the dynamical systems approach (Ioannou, Chien 1993; Swaroop et al. 1994).

Substituting $e=v(\rho)-v$ in Equation (5) and re-arranging resulted in:

$$
\frac{d(v(\rho))}{d \rho} \cdot \frac{d \rho}{d t}-\frac{d v}{d t}=-a \cdot(v(\rho)-v) .
$$

Discretizing Equation (6) using a time step $h$ resulted in:

$$
\begin{aligned}
& \frac{d(v(\rho))}{d \rho} \cdot \frac{(\rho(k+1)-\rho(k))}{h}- \\
& \frac{(v(k+1)-v(k))}{h}=-a \cdot(v(\rho)-v) .
\end{aligned}
$$

Substituting:

$\frac{\rho(k+1)-\rho(k)}{h}=\frac{1}{L}\left(q_{\text {en }}(k)-\rho(k) \cdot v(k)+q_{\text {side }}(k)\right)$ from Equation (4), the dynamic speed equation (that is, the equation governing the evolution of speed) can be obtained as:

$$
\begin{aligned}
& v(k+1)=v(k)+a \cdot h \cdot(v(\rho)-v(k))+\frac{h}{L} \times \\
& \frac{d(v(\rho))}{d \rho} \cdot\left(q_{e n}(k)-\rho(k) \cdot v(k)+q_{\text {side }}(k)\right) .
\end{aligned}
$$

The general formulation of the lumped parameter model is represented by Equations (4) and (5). Here the heterogeneity of traffic was not explicitly considered. The site specific speed density relationship $v(\rho)$ was also developed without considering heterogeneity and incorporated in Equation (8). A brief description of the developed speed density relation and the details on incorporating it in Equation (8) is provided below.

Based on the field data collected from locations along the study stretch on Rajiv Gandhi road in Chennai, India, the best fitting speed density relation was identified empirically (base work of this can be found at Thankappan, Vanajakshi (2012)). To take into account the lack of lane discipline, the roadway was analyzed without considering the traffic lanes and hence flow was expressed in [veh/hr] and density in [veh/km]. Using this data, the best fitting traffic stream model was found to be a two-regime relationship of the form:

$$
\left\{\begin{array}{l}
v=v_{f} \cdot \exp \left(-0.5 \cdot\left(\frac{\rho}{\rho_{c r}}\right)^{2}\right) \text { for } 0 \leq \rho \leq \rho_{c r} ; \\
v=C \cdot\left(\frac{\rho_{j}}{\rho}-1\right) \text { for } \rho_{c r} \leq \rho \leq \rho_{j} .
\end{array}\right.
$$


where: $v_{f}$ is the free flow speed; $\rho_{c r}$ indicates the critical density (density at maximum flow); $\rho_{j}$ represents the jam density and $C$ is a model parameter.

A plot of the predicted values from this stream model against the observed values from field is shown in Fig. 2. Values of the model parameters are shown in Table 1 .

Incorporating Equation (9) in Equation (8), the dynamic speed equation reduced to:

$$
\begin{cases}v(k+1)=v(k)+\operatorname{comp}_{1} & \text { for } 0 \leq \rho(k) \leq \rho_{c r} \\ v(k+1)=v(k)+\operatorname{comp}_{2} & \text { for } \rho_{c r} \leq \rho(k) \leq \rho_{j}\end{cases}
$$

where:

$$
\begin{aligned}
& \operatorname{comp} p_{1}=a \cdot h \cdot\left(v_{f} \cdot \exp \left(-0.5 \cdot\left(\frac{\rho(k)}{\rho_{c r}}\right)^{2}\right)-v(k)\right)- \\
& \frac{v_{f} \cdot h \cdot \rho(k)}{\left(\rho_{c r}\right)^{2} \cdot L} \cdot \exp \left(-0.5 \cdot\left(\frac{\rho(k)}{\rho_{c r}}\right)^{2}\right) \times \\
& \left(q_{e n}(k)-\rho(k) \cdot v(k) \cdot q_{\text {side }}(k)\right) ; \\
& \text { comp } p_{2}=a \cdot h \cdot\left(C\left(\frac{\rho_{j}}{\rho(k)}-1\right)-v(k)\right)- \\
& \frac{C \cdot \rho_{j} \cdot h}{L \cdot(\rho(k))^{2}} \cdot\left(q_{\text {en }}(k)-\rho(k) \cdot v(k)+q_{\text {side }}(k)\right) .
\end{aligned}
$$

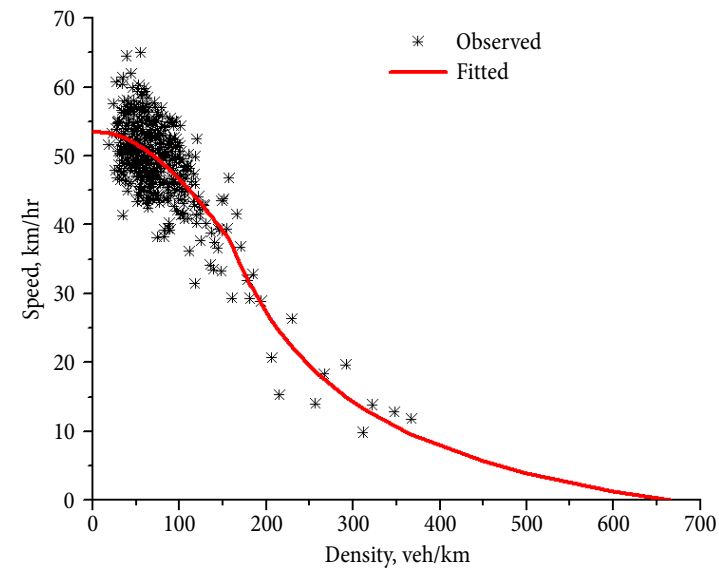

Fig. 2. Plot of developed stream model against the observed values from field
Thus, the complete model for traffic without considering heterogeneity can be represented by Equation (4) and Equation (10).

\section{Incorporation of Heterogeneity into the Model}

The above model was subsequently modified to incorporate heterogeneity in different ways as explained below.

\subsection{Use of Static Passenger Car Units}

One common way to consider different categories of vehicles in a traffic stream is to convert them into a homogeneous equivalent using either static values of PCU or dynamic values of PCU and this approach has been applied in this study. The static values of PCU recommended by Indian Road Congress (IRC 1990) were used first to convert the heterogeneous traffic into a homogeneous equivalent. Thus, in Equations (4) and (8) traffic flow was considered in [PCU/hr] and traffic density in $[\mathrm{PCU} / \mathrm{km}]$. The site specific speed density relationship $v(\rho)$ developed using the data converted into a common unit using static values of PCU was incorporated in Equation (8). During development of the equation for $v(\rho)$ in this case, the data were measured separately for different categories of vehicles and then converted into static PCU units. Three vehicle groups were considered: Two Wheelers (TWs), Three Wheelers (ThWs) and Four Wheelers (FWs). TWs included motorcycles, scooters and mopeds, ThWs included auto-rickshaws and small three wheeled tempos, and FWs included light passenger cars and heavy commercial vehicles. Based on availability of data, the model can be extended easily to incorporate more classes. Table 2 presents the values of PCU recommended by Indian Roads Congress (IRC 1990) that have been used in this study (IRC 1990). The average traffic composition of different vehicles present in the study stretch were observed to be around $51 \%$, $7 \%, 34 \%, 6 \%$ and $2 \%$ of TWs, ThWs (auto rickshaw), passenger cars, light commercial vehicles and heavy commercial vehicles (buses and trucks) respectively. In

\begin{tabular}{|c|c|c|c|c|c|}
\hline \multirow{2}{*}{ Category } & & \multicolumn{4}{|c|}{ Parameters } \\
\hline & & $v_{f}[\mathrm{~km} / \mathrm{hr}]$ & $\rho_{c r}$ & $\rho_{j}$ & $C[\mathrm{~km} / \mathrm{hr}]$ \\
\hline Data without considering heterogeneity & & 53.58 & $157 \mathrm{veh} / \mathrm{km}$ & $665 \mathrm{veh} / \mathrm{km}$ & 11.74 \\
\hline Data converted in to static PCU units & & 53.86 & $149 \mathrm{PCU} / \mathrm{km}$ & $602 \mathrm{PCU} / \mathrm{km}$ & 12.15 \\
\hline Data converted in to dynamic TWU units & & 52.54 & $600 \mathrm{TWU} / \mathrm{km}$ & $5800 \mathrm{TWU} / \mathrm{km}$ & 4.63 \\
\hline \multirow{3}{*}{ Classified data without considering interaction } & Class 1 & 51.93 & $97 \mathrm{TW} / \mathrm{km}$ & $375 \mathrm{TW} / \mathrm{km}$ & 12.06 \\
\hline & Class 2 & 41.56 & $15 \mathrm{ThW} / \mathrm{km}$ & $49 \mathrm{ThW} / \mathrm{km}$ & 15.17 \\
\hline & Class 3 & 57.30 & $63 \mathrm{FW} / \mathrm{km}$ & $250 \mathrm{FW} / \mathrm{km}$ & 12.58 \\
\hline \multirow{3}{*}{ Classified data with considering interaction } & Class 1 & 52.58 & \multirow{3}{*}{$157 \mathrm{veh} / \mathrm{km}$} & \multirow{3}{*}{$674 \mathrm{veh} / \mathrm{km}$} & 11.94 \\
\hline & Class 2 & 43.24 & & & 9.83 \\
\hline & Class 3 & 57.72 & & & 11.04 \\
\hline
\end{tabular}
this study, a weighted average value of PCU was calculated for FWs, considering the proportion of different categories of vehicles coming under the category of FWs as the weights. The functional forms of the speed-den-

Table 1. Parameters of the derived steady-state speed-density relationships 
Table 2. Recommended PCU values of vehicles on urban roads by IRC (1990)

\begin{tabular}{|c|c|c|}
\hline \multirow{3}{*}{ Vehicle type } & \multicolumn{2}{|c|}{ Equivalent PCU values } \\
\hline & \multicolumn{2}{|c|}{$\begin{array}{l}\text { Percentage composition of vehicle } \\
\text { type in traffic stream }\end{array}$} \\
\hline & $5 \%$ & $10 \%$ and above \\
\hline \multicolumn{3}{|l|}{ Fast Vehicles: } \\
\hline $\begin{array}{l}\text { 1. Two Wheeler Motor } \\
\text { Cycle or Scooter }\end{array}$ & 0.5 & 0.75 \\
\hline $\begin{array}{l}\text { 2. Passenger Car, } \\
\text { Pick-up Van }\end{array}$ & 1.0 & 1.0 \\
\hline 3. Auto-rickshaw & 1.2 & 2.0 \\
\hline $\begin{array}{l}\text { 4. Light Commercial } \\
\text { Vehicle }\end{array}$ & 1.4 & 1.2 \\
\hline 5. Truck or Bus & 2.2 & 3.7 \\
\hline $\begin{array}{l}\text { 6. Agricultural Tractor } \\
\text { Trailer }\end{array}$ & 4.0 & 5.0 \\
\hline \multicolumn{3}{|l|}{ Slow Vehicles: } \\
\hline 1. Cycle & 0.4 & 0.5 \\
\hline 2. Cycle Rickshaw & 1.5 & 2.0 \\
\hline $\begin{array}{l}\text { 3. Tonga (Horse } \\
\text { Drawn Vehicle) }\end{array}$ & 1.5 & 2.0 \\
\hline 4. Hand Cart & 2.0 & 3.0 \\
\hline
\end{tabular}

sity relationship and dynamic speed equation for this PCU converted data were obtained similar to the case without considering heterogeneity. Various parameters associated with this relationship are shown in Table 1.

Thus, the complete model formulation after incorporating static PCU was represented by Equation (4) and Equation (10) with flow rates and density expressed in $[\mathrm{PCU} / \mathrm{hr}]$ and $[\mathrm{PCU} / \mathrm{km}]$ respectively and with the parameters developed using the PCU converted data.

\subsection{Use of Dynamic Two Wheeler Units}

The next approach was the use of dynamic values of equivalents to aggregate the heterogeneous traffic into a homogeneous one, since the equivalency factor for a vehicle type is not a static factor as is usually assumed (Chandra et al. 1995). The dynamic values of the equivalency factors used in this study were developed based on the formula for dynamic PCU proposed by Chandra et al. (1995), which is considered to be most suitable for mixed traffic conditions in India (Chandra, Kumar 2003). According to Chandra et al. (1995), the PCU value for different vehicles under mixed traffic situation is directly proportional to the speed ratio and inversely proportional to the space occupancy ratio with respect to a car, which is taken as the reference vehicle. Thus,

$$
\mathrm{PCU}_{i}=\frac{\left(\frac{v_{c}}{v_{i}}\right)}{\left(\frac{A_{c}}{A_{i}}\right)},
$$

where: $\mathrm{PCU}_{i}$ indicates the dynamic passenger car unit for the $i$ th category of vehicle; $v_{c} / v_{i}$ is the speed ratio for the car to the $i$ th vehicle; $A_{c} / A_{i}$ is space ratio for the car to the $i$ th vehicle.

In the present study, the motorized two wheeler is considered as the reference vehicle, since the proportion of two wheelers is observed to be more than $50 \%$ of the composition in the study stretch. Hence, Equation (11) was modified to incorporate this change and the formula for calculating the dynamic TWU values was obtained as:

$$
\mathrm{TWU}_{i}=\frac{\left(\frac{v_{\mathrm{TW}}}{v_{i}}\right)}{\left(\frac{A_{\mathrm{TW}}}{A_{i}}\right)},
$$

where: $\mathrm{TWU}_{i}$ indicates the dynamic TWU for the $i$ th category of vehicle; $v_{W} / v_{i}$ is the speed ratio for (Two Wheelers) TW to the $i$ th vehicle; $A_{W} / A_{i}$ is space ratio for TW to the $i$ th vehicle. The vehicle dimensions for calculating the space ratios for the different categories of vehicles were adopted from an earlier study conducted in Chennai (Arasan, Krishnamurthy 2008). For the FW category, weighted average values of area were used using the proportions of different categories of vehicles coming under this category as weights.

Equations (4) and (8) were modified to incorporate the heterogeneity in terms of this dynamic TWU. The governing equation for density (Equation (4)) was modified by expressing the flow rates in terms of the dynamic TWU equivalents indicated by Equation (12) and was obtained as:

$$
\begin{aligned}
& \rho(\mathrm{k}+1)=\rho(k)+\frac{h}{L} \times \\
& q_{\text {en }}^{1}(k)+q_{\text {en }}^{2}(k) \cdot \frac{\left(\frac{v_{1}(k)}{v_{2}(k)}\right)}{\left(\frac{A_{1}}{A_{2}}\right)}+ \\
& q_{\text {en }}^{3}(k) \cdot \frac{\left(\frac{v_{1}(k)}{v_{3}(k)}\right)}{\left(\frac{A_{1}}{A_{3}}\right)}-\rho(k) \cdot v(k)+q_{\text {side }}^{1}(k)+ \\
& \left.q_{\text {side }}^{2}(k) \cdot \frac{\left(\frac{v_{1}(k)}{v_{2}(k)}\right)}{\left(\frac{A_{1}}{A_{2}}\right)}+q_{\text {eside }}^{3}(k) \cdot \frac{\left(\frac{v_{1}(k)}{v_{3}(k)}\right)}{\left(\frac{A_{1}}{A_{3}}\right)}\right) .
\end{aligned}
$$

The dynamic equation for speed (Equation (8)) was modified by incorporating the steady-state speed-density relationship derived from the empirical data converted using the dynamic TWU units. This relationship was also obtained in a similar form as indicated in Equation (9), where $\rho$ is in $[\mathrm{TWU} / \mathrm{km}]$. The corresponding parameters obtained are shown in Table 1. 
This derived speed-density relationship was incorporated in Equation (8) to get the dynamic equation for speed in this case and was obtained as:

$$
\begin{aligned}
& \left\{\begin{array}{l}
v(k+1)=v(k)+\operatorname{comp}_{1} \text { for } 0 \leq \rho(k) \leq \rho_{c r} \\
v(k+1)=v(k)+\operatorname{comp}_{2} \text { for } \rho_{c r} \leq \rho(k) \leq \rho_{j}
\end{array}\right. \\
& \operatorname{comp}_{1}=a \cdot h \cdot\left(v_{f} \cdot \exp \left(-0.5 \cdot\left(\frac{\rho(k)}{\rho_{c r}}\right)^{2}\right)-v(k)\right)- \\
& \frac{v_{f} \cdot h \cdot \rho(k)}{\rho_{c r}^{2} \cdot L} \cdot \exp \left(-0.5\left(\frac{\rho(k)}{\rho_{c r}}\right)^{2}\right) \times \\
& \left(q_{e n}^{1}(k)+q_{e n}^{2}(k) \cdot \frac{\left(\frac{v_{1}(k)}{v_{2}(k)}\right)}{\left(\frac{A_{1}}{A_{2}}\right)}+q_{e n}^{3}(k) \cdot \frac{\left(\frac{v_{1}(k)}{v_{3}(k)}\right)}{\left(\frac{A_{1}}{A_{3}}\right)}-\right. \\
& \rho(k) \cdot v(k)+q_{\text {side }}^{1}(k)+q_{\text {side }}^{2}(k) \times \\
& \left.\frac{\left(\frac{v_{1}(k)}{v_{2}(k)}\right)}{\left(\frac{A_{1}}{A_{2}}\right)}+q_{\text {side }}^{3}(k) \cdot \frac{\left(\frac{v_{1}(k)}{v_{3}(k)}\right)}{\left(\frac{A_{1}}{A_{3}}\right)}\right) \text {; } \\
& \operatorname{comp}_{2}=a \cdot h \cdot\left(C \cdot\left(\frac{\rho_{j}}{\rho(k)}-1\right)-v(k)\right)- \\
& \frac{C \cdot \rho_{j} \cdot h}{L \cdot(\rho(k))^{2}} \cdot\left(q_{e n}^{1}(k)+q_{e n}^{2}(k) \cdot \frac{\left(\frac{v_{1}(k)}{v_{2}(k)}\right)}{\left(\frac{A_{1}}{A_{2}}\right)}+\right. \\
& q_{\text {en }}^{3}(k) \cdot \frac{\left(\frac{v_{1}(k)}{v_{3}(k)}\right)}{\left(\frac{A_{1}}{A_{3}}\right)}-\rho(k) \cdot v(k)+q_{\text {side }}^{1}(k)+ \\
& \left.q_{\text {side }}^{2}(k) \cdot \frac{\left(\frac{v_{1}(k)}{v_{2}(k)}\right)}{\left(\frac{A_{1}}{A_{2}}\right)}+q_{\text {side }}^{3}(k) \cdot \frac{\left(\frac{v_{1}(k)}{v_{3}(k)}\right)}{\left(\frac{A_{1}}{A_{3}}\right)}\right) .
\end{aligned}
$$

principle and was obtained as:

$$
\begin{aligned}
& \rho_{1}(k+1)=\rho_{1}(k)+\frac{h}{L} \times \\
& \left(q_{\text {en }}^{1}(k)-\rho_{1}(k) \cdot v_{1}(k)+q_{\text {side }}^{1}(k)\right) ; \\
& \rho_{2}(k+1)=\rho_{2}(k)+\frac{h}{L} \times \\
& \left(q_{\text {en }}^{2}(k)-\rho_{2}(k) \cdot v_{2}(k)+q_{\text {side }}^{2}(k)\right) ; \\
& \rho_{3}(k+1)=\rho_{3}(k)+\frac{h}{L} \times \\
& \left(q_{\text {en }}^{3}(k)-\rho_{3}(k) \cdot v_{3}(k)+q_{\text {side }}^{3}(k)\right) .
\end{aligned}
$$

The dynamic equations for space-mean speed of the three classes of vehicles were formulated using the steady state speed-density relationships developed for these three classes. Two different approaches were followed in this study while developing the classified steady state speed-density relationships. In the first approach of this multi-class modeling, the speed of a particular class of vehicle is assumed as a function of density of that particular class alone and separate speed-density relations were developed for all the three classes (corresponding parameters are shown in Table 1) and were obtained as: $\left\{\begin{array}{l}v_{1}=v_{f}(1) \cdot \exp \left(-0.5 \cdot\left(\frac{\rho_{1}}{\rho_{c r}(1)}\right)^{2}\right) \text { for } 0 \leq \rho_{1} \leq \rho_{c r}(1) \\ v_{1}=C_{1} \cdot\left(\frac{\rho_{j}(1)}{\rho_{1}}-1\right) \text { for } \rho_{c r}(1) \leq \rho_{1} \leq \rho_{j}(1)\end{array}\right.$

$\left\{\begin{array}{l}v_{2}=v_{f}(2) \cdot \exp \left(-0.5 \cdot\left(\frac{\rho_{2}}{\rho_{c r}(2)}\right)^{2}\right) \text { for } 0 \leq \rho_{2} \leq \rho_{c r}(2) \\ v_{2}=C_{2} \cdot\left(\frac{\rho_{j}(2)}{\rho_{2}}-1\right) \text { for } \rho_{c r}(2) \leq \rho_{2} \leq \rho_{j}(2)\end{array}\right.$ $\left\{\begin{array}{l}v_{3}=v_{f}(3) \cdot \exp \left(-0.5 \cdot\left(\frac{\rho_{3}}{\rho_{c r}(3)}\right)^{2}\right) \text { for } 0 \leq \rho_{3} \leq \rho_{c r}(3) ; \\ v_{3}=C_{3} \cdot\left(\frac{\rho_{j}(3)}{\rho_{3}}-1\right) \text { for } \rho_{c r}(3) \leq \rho_{3} \leq \rho_{j}(3) .\end{array}\right.$

Using these equations, the dynamic speed equations were formulated for TWs, ThWs and FWs were obtained as:

$$
\begin{cases}v_{1}(k+1)=v_{1}(k)+\operatorname{comp}_{1} & \text { for } 0 \leq \rho_{1}(k) \leq \rho_{c r}(1) \\ v_{1}(k+1)=v_{1}(k)+\operatorname{comp}_{2} & \text { for } \rho_{c r}(1) \leq \rho_{1}(k) \leq \rho_{j}(1),\end{cases}
$$

A third way of introducing heterogeneity in traffic flow is to consider different classes of vehicles separately in the modeling process. The classification considered in this study was three vehicle groups namely, TWs (class 1), ThWs (class 2) and FWs (class 3) as mentioned earlier. The governing equation for density was modified by incorporating separate state equations for the three different classes of vehicles based on the conservation where:

$$
\begin{aligned}
& \operatorname{comp} p_{1}=b \cdot h \cdot\left(v_{f}(1) \cdot \exp \left(-0.5 \cdot\left(\frac{\rho_{1}(k)}{\rho_{c r}(1)}\right)^{2}\right)-v_{1}(k)\right)- \\
& \frac{v_{f}(1) \cdot h \cdot \rho_{1}(k)}{\left(\rho_{c r}(1)\right)^{2} \cdot L} \cdot \exp \left(-0.5 \cdot\left(\frac{\rho_{1}(k)}{\rho_{c r}(1)}\right)^{2}\right) \times
\end{aligned}
$$




$$
\begin{aligned}
& \left(q_{\text {en }}^{1}(k)-\rho_{1}(k) \cdot v_{1}(k)+q_{\text {side }}^{1}(k)\right) ; \\
& \text { comp } p_{2}=b \cdot h \cdot\left(C_{1}\left(\frac{\rho_{j}(1)}{\rho_{1}(k)}-1\right)-v_{1}(k)\right)-\frac{C_{1} \cdot \rho_{j}(1) \cdot h}{L \cdot\left(\rho_{1}(k)\right)^{2}} \times \\
& \left(q_{\text {en }}^{1}(k)-\rho_{1}(k) \cdot v_{1}(k)+q_{\text {side }}^{1}(k)\right) . \\
& \begin{cases}v_{2}(k+1)=v_{2}(k)+c o m p_{1} \text { for } 0 \leq \rho_{2}(k) \leq \rho_{c r}(2) ; \\
v_{2}(k+1)=v_{2}(k)+c o m p_{2} \text { for } \rho_{c r}(2) \leq \rho_{2}(k) \leq \rho_{j}(2),\end{cases}
\end{aligned}
$$

where:

$$
\begin{aligned}
& \text { where: } \\
& \text { comp } p_{1}=b \cdot h \cdot\left(v_{f}(2) \cdot \exp \left(-0.5 \cdot\left(\frac{\rho_{2}(k)}{\rho_{c r}(2)}\right)^{2}\right)-v_{2}(k)\right)- \\
& \frac{v_{f}(2) \cdot h \cdot \rho_{2}(k)}{\left(\rho_{c r}(2)\right)^{2} \cdot L} \cdot \exp \left(-0.5 \cdot\left(\frac{\rho_{2}(k)}{\rho_{c r}(2)}\right)^{2}\right) \times \\
& \left(q_{e n}^{2}(k)-\rho_{2}(k) \cdot v_{2}(k)+q_{s i d e}^{2}(k)\right) ; \\
& \text { comp } p_{2}=b \cdot h \cdot\left(C_{2} \cdot\left(\frac{\rho_{j}(2)}{\rho_{2}(k)}-1\right)-v_{2}(k)\right)- \\
& \frac{C_{2} \cdot \rho_{j}(2) \cdot h}{L \cdot\left(\rho_{2}(k)\right)^{2}} \cdot\left(q_{e n}^{2}(k)-\rho_{2}(k) \cdot v_{2}(k)+q_{s i d e}^{2}(k)\right) . \\
& \left\{\begin{array}{l}
v_{3}(k+1)=v_{3}(k)+c o m p_{1} \text { for } 0 \leq \rho_{3}(k) \leq \rho_{c r}(3) ; \\
v_{3}(k+1)=v_{3}(k)+c o m p_{2} \text { for } \rho_{c r}(3) \leq \rho_{3}(k) \leq \rho_{j}(3),
\end{array}\right.
\end{aligned}
$$

where:

$$
\begin{aligned}
& \text { where: } \\
& \text { comp } p_{1}=b \cdot h \cdot\left(v_{f}(3) \cdot \exp \left(-0.5\left(\frac{\rho_{3}(k)}{\rho_{c r}(3)}\right)^{2}\right)-v_{3}(k)\right)- \\
& \frac{v_{f}(3) \cdot h \cdot \rho_{3}(k)}{\left(\rho_{c r}(3)\right)^{2} \cdot L} \cdot \exp \left(-0.5\left(\frac{\rho_{3}(k)}{\rho_{c r}(3)}\right)^{2}\right) \times \\
& \left(q_{e n}^{3}(k)-\rho_{3}(k) \cdot v_{3}(k)+q_{\text {side }}^{3}(k)\right) ; \\
& \text { comp } p_{2}=b \cdot h \cdot\left(C_{3}\left(\frac{\rho_{j}(3)}{\rho_{3}(k)}-1\right)-v_{3}(k)\right)-\frac{C_{3} \cdot \rho_{j}(3) \cdot h}{L \cdot\left(\rho_{3}(k)\right)^{2}} \times \\
& \left(q_{e n}^{3}(k)-\rho_{3}(k) \cdot v_{3}(k)+q_{\text {side }}^{3}(k)\right) .
\end{aligned}
$$

Thus, the complete model based on this multi-class modeling can be represented by Equations (15) to (17) and Equations (21) to (23).

\subsection{Multi-Class Model with Interaction}

For the multi-class stream model, it is assumed that the movement of one category of vehicle is influenced by the presence of all other vehicles. Hence, the speed of one category of vehicle is assumed as a function of total density $\rho$. This is a more realistic representation under In- dian traffic conditions than the previous approach, since vehicles of different categories typically move without segregation on Indian roads. The corresponding speeddensity relationships (parameters shown in Table 1) developed for the three classes were obtained as:

$$
\begin{aligned}
& \left\{\begin{array}{l}
v_{1}=v_{f}(1) \cdot \exp \left(-0.5 \cdot\left(\frac{\rho}{\rho_{c r}}\right)^{2}\right) \text { for } 0 \leq \rho \leq \rho_{c r} ; \\
v_{1}=C_{1} \cdot\left(\frac{\rho_{j}}{\rho}-1\right) \text { for } \rho_{c r} \leq \rho \leq \rho_{j} ; \\
v_{2}=v_{f}(2) \cdot \exp \left(-0.5\left(\frac{\rho}{\rho_{c r}}\right)^{2}\right) \text { for } 0 \leq \rho \leq \rho_{c r} ; \\
v_{2}=C_{2} \cdot\left(\frac{\rho_{j}}{\rho}-1\right) \text { for } \rho_{c r} \leq \rho \leq \rho_{j} ; \\
v_{3}=v_{f}(3) \cdot \exp \left(-0.5\left(\frac{\rho}{\rho_{c r}}\right)^{2}\right) \text { for } 0 \leq \rho \leq \rho_{c r} ; \\
v_{3}=C_{3} \cdot\left(\frac{\rho_{j}}{\rho}-1\right) \text { for } \rho_{c r} \leq \rho \leq \rho_{j} \cdot
\end{array}\right.
\end{aligned}
$$

Using these equations, the governing equation for the space mean speed of each class of vehicles was formulated based on the methodology as before and the equations were obtained as:

$\begin{cases}v_{1}(k+1)=v_{1}(k)+\operatorname{comp}_{1} & \text { for } 0 \leq \rho(k) \leq \rho_{c r} ; \\ v_{1}(k+1)=v_{1}(k)+\operatorname{comp}_{2} & \text { for } \rho_{c r} \leq \rho(k) \leq \rho_{j}\end{cases}$

where:

$\operatorname{comp} p_{1}=b \cdot h \cdot\left(v_{f}(1) \cdot \exp \left(-0.5\left(\frac{\rho(k)}{\rho_{c r}}\right)^{2}\right)-v_{1}(k)\right)-$ $\frac{v_{f}(1) \cdot h \cdot \rho(k)}{\left(\rho_{c r}\right)^{2} \cdot L} \cdot\left(-0.5\left(\frac{\rho(k)}{\rho_{c r}}\right)^{2}\right) \times$

$\left(q_{\text {en }}^{1}(k)+q_{\text {en }}^{2}(k)+q_{\text {en }}^{3}(k)-\rho_{1}(k) \cdot v_{1}(k)-\rho_{2}(k) \cdot v_{2}(k)-\right.$ $\left.\rho_{3}(k) \cdot v_{3}(k)+q_{\text {side }}^{1}(k)+q_{\text {side }}^{2}(k)+q_{\text {side }}^{3}(k)\right)$;

$\operatorname{comp}_{2}=b \cdot h \cdot\left(C_{1} \cdot\left(\frac{\rho_{j}}{\rho(k)}-1\right)-v_{1}(k)\right)-\frac{C_{1} \cdot \rho_{j} \cdot h}{L \cdot(\rho(k))^{2}} \times$

$\left(q_{e n}^{1}(k)+q_{e n}^{2}(k)+q_{e n}^{3}(k)-\rho_{1}(k) \cdot v_{1}(k)-\rho_{2}(k) \cdot v_{2}(k)-\right.$

$\left.\rho_{3}(k) \cdot v_{3}(k)+q_{\text {side }}^{1}(k)+q_{\text {side }}^{2}(k)+q_{\text {side }}^{3}(k)\right)$.

$\begin{cases}v_{2}(k+1)=v_{2}(k)+c o m p_{1} & \text { for } 0 \leq \rho(k) \leq \rho_{c r} ; \\ v_{2}(k+1)=v_{2}(k)+c o m p_{2} & \text { for } \rho_{c r} \leq \rho(k) \leq \rho_{j}\end{cases}$

where:

$c o m p_{1}=b \cdot h \cdot\left(v_{f}(2) \cdot \exp \left(-0.5 \cdot\left(\frac{\rho(k)}{\rho_{c r}}\right)^{2}\right)-v_{2}(k)\right)-$ 


$$
\begin{aligned}
& \frac{v_{f}(2) \cdot h \cdot \rho(k)}{\left(\rho_{c r}\right)^{2} \cdot L} \cdot\left(-0.5\left(\frac{\rho(k)}{\rho_{c r}}\right)^{2}\right) \times \\
& \left(q_{\text {en }}^{1}(k)+q_{\text {en }}^{2}(k)+q_{\text {en }}^{3}(k)-\rho_{1}(k) \cdot v_{1}(k)-\rho_{2}(k) \cdot v_{2}(k)-\right. \\
& \left.\rho_{3}(k) \cdot v_{3}(k)+q_{\text {side }}^{1}(k)+q_{\text {side }}^{2}(k)+q_{\text {side }}^{3}(k)\right) ; \\
& \text { comp } p_{2}=b \cdot h \cdot\left(C_{2}\left(\frac{\rho_{j}}{\rho(k)}-1\right)-v_{2}(k)\right)-\frac{C_{2} \cdot \rho_{j} \cdot h}{L \cdot(\rho(k))^{2}} \times \\
& \left(q_{\text {en }}^{1}(k)+q_{\text {en }}^{2}(k)+q_{\text {en }}^{3}(k)-\rho_{1}(k) \cdot v_{1}(k)-\rho_{2}(k) \cdot v_{2}(k)-\right. \\
& \left.\rho_{3}(k) \cdot v_{3}(k)+q_{\text {side }}^{1}(k)+q_{\text {side }}^{2}(k)+q_{\text {side }}^{3}(k)\right) \cdot \\
& \left\{\begin{array}{l}
v_{3}(k+1)=v_{3}(k)+\text { comp } p_{1} \quad \text { for } 0 \leq \rho(k) \leq \rho_{c r} ; \\
v_{3}(k+1)=v_{3}(k)+c o m p_{2} \quad \text { for } \rho_{c r} \leq \rho(k) \leq \rho_{j}, \quad \text { (29) }
\end{array}\right.
\end{aligned}
$$

where:

$$
\begin{aligned}
& \text { where: } \\
& \text { comp } p_{1}=b \cdot h \cdot\left(v_{f}(3) \cdot \exp \left(-0.5 \cdot\left(\frac{\rho(k)}{\rho_{c r}}\right)^{2}\right)-v_{3}(k)\right)- \\
& \frac{v_{f}(3) \cdot h \cdot \rho(k)}{\left(\rho_{c r}\right)^{2} \cdot L} \cdot\left(-0.5 \cdot\left(\frac{\rho(k)}{\rho_{c r}}\right)^{2}\right) \times \\
& \left(q_{\text {en }}^{1}(k)+q_{\text {en }}^{2}(k)+q_{\text {en }}^{3}(k)-\rho_{1}(k) \cdot v_{1}(k)-\rho_{2}(k) \cdot v_{2}(k)-\right. \\
& \left.\rho_{3}(k) \cdot v_{3}(k)+q_{\text {side }}^{1}(k)+q_{\text {side }}^{2}(k)+q_{\text {side }}^{3}(k)\right) ; \\
& \text { comp } p_{2}=b \cdot h \cdot\left(C_{3}\left(\frac{\rho_{j}}{\rho(k)}-1\right)-v_{3}(k)\right)-\frac{C_{3} \cdot \rho_{j} \cdot h}{(\rho(k))^{2} \cdot L} \times \\
& \left(q_{\text {en }}^{1}(k)+q_{\text {en }}^{2}(k)+q_{\text {en }}^{3}(k)-\rho_{1}(k) \cdot v_{1}(k)-\rho_{2}(k) \cdot v_{2}(k)-\right. \\
& \left.\rho_{3}(k) \cdot v_{3}(k)+q_{\text {side }}^{1}(k)+q_{\text {side }}^{2}(k)+q_{\text {side }}^{3}(k)\right) \cdot
\end{aligned}
$$

Equations (15) to (17), along with Equations (27) to (29) represent the complete model based on the multiclass modeling with interaction.

It can be seen that in all these models presented above, the generic governing equations are derived using the conservation of vehicles and the hypothesis regarding the evolution of the error between the speed values estimated using the steady state relation and the observed values from field. This will hold good for any road segment. However, the specific equations for the evolution of error obtained from the developed steadystate speed-density relation (traffic stream models) are site specific (Hall 2001). Thus, though the generic equations are transferable, the site specific stream model is transferable only to sections with similar characteristics. In other cases, the section specific stream model should be known or developed and needs to be used in the generic governing equations for good performance.

\section{Model-Based Estimation Scheme}

The Kalman filter (Kalman 1960) is an optimal estimator suitable for recursive estimation and prediction of variables that characterize dynamical systems. It is a modelbased scheme and it incorporates the stochastic nature of the process disturbance and the measurement noise. The process disturbance and the measurement noise are assumed to be independent of one another, white and normally distributed with zero mean. The Kalman filter works like a predictor corrector algorithm, i.e., it first predicts an 'a priori' estimate of the state variables using the system model, the system inputs and the state estimate from the previous time interval, and then corrects the same using measurements to obtain an 'a posteriori' state estimate. The Kalman filter is used for estimation and prediction when the governing equations of the system are linear. When the governing equations are nonlinear, the Extended Kalman Filter (EKF) is commonly used (Maybeck 1979, 1982a, 1982b). The EKF linearizes the governing equations at each time step about the estimate obtained from the previous time step. The Kalman filter and the EKF have been widely used in many disciplines including the field of transportation (Nahi, Trivedi 1973; Gazis, Szeto 1974; Okutani, Stephanedes 1984; Nanthawichit et al. 2003; Wang, Papageorgiou 2005; Padiath et al. 2009, 2010; Anand et al. 2011).

In the present study, as the models developed are non-linear, the EKF is used for the purpose of estimating traffic density. Combining the model presented in the previous section and using the EKF, the model based estimation scheme was designed as detailed below.

Considering a non-linear system whose model is given by:

$$
\begin{aligned}
& \mathbf{x}_{k+1}=\mathbf{f}\left(\mathbf{x}_{k}, \mathbf{u}_{k}, \mathbf{w}_{k}\right) ; \\
& \mathbf{z}_{k}=\mathbf{g}\left(\mathbf{x}_{k}, \mathbf{v}_{k}\right),
\end{aligned}
$$

where: $\mathbf{x}_{k}$ is the system state; $\mathbf{z}_{k}$ is the system output; $\mathbf{u}_{k}$ is the system input; $\mathbf{w}_{k}$ is the process disturbance; $\mathbf{v}_{k}$ is the measurement noise at the $k$ th instant of time. Here, f represents the non-linear function that relates the state at time step $k$ to the state at time step $k+1$. Similarly $\mathbf{g}$ is the non-linear function that relates the state to the measurement. The above equations can be linearized using Taylor's Series expansion to result in:

$$
\begin{aligned}
& \mathbf{x}_{k+1}=\tilde{\mathbf{x}}_{k+1}+\mathbf{A}\left(\mathbf{x}_{k}-\hat{\mathbf{x}}_{k}^{+}\right)+\mathbf{W} \mathbf{w}_{k} \\
& \mathbf{z}_{k}=\tilde{\mathbf{z}}_{k}+\mathbf{H}\left(\mathbf{x}_{k}-\tilde{\mathbf{x}}_{k}\right)+\mathbf{V} \mathbf{v}_{k} \\
& \tilde{\mathbf{x}}_{k+1}=\mathbf{f}\left(\hat{\mathbf{x}}_{k}^{+}, \mathbf{u}_{k}, 0\right) \\
& \tilde{\mathbf{z}}_{k}=\mathbf{g}\left(\tilde{\mathbf{x}}_{k}, 0\right)
\end{aligned}
$$

where: $\tilde{\mathbf{x}}, \tilde{\mathbf{z}}$ are the approximate state and measurement variables without considering the process disturbance and measurement noise as indicated by Equations (34) and (35) respectively in which $\hat{\mathbf{x}}_{k}{ }^{+}$is the a posteriori estimate of the state (from a previous time step $k$ ); $\mathbf{A}$ is the matrix of the partial derivative of $\mathbf{f}$ with respect to $\mathbf{x}$; $\mathbf{W}$ is the matrix of the partial derivative of $\mathbf{f}$ with respect to $\mathbf{w} ; \mathbf{H}$ is the matrix of the partial derivative of $\mathbf{g}$ with respect to $\mathbf{x}$ and $\mathbf{V}$ is the matrix of the partial derivative of $\mathbf{g}$ with respect to $\mathbf{v}$.

Let $\mathbf{Q}$ denote the process disturbance covariance, $\mathbf{R}$ denote the measurement noise covariance, $\hat{\mathbf{x}}_{k}^{-}$denote the $a$ priori estimate of the state variables at the $k$ th instant of time and $\mathbf{P}_{k}^{-}$and $\mathbf{P}_{k}^{+}$denote respectively the $a$ 
priori and the a posteriori error covariance at the $k$ th instant of time.

Now the following steps were followed recursively for estimation using EKF:

1. The a priori estimate in the $(k+1)$ th interval of time was obtained through:

$$
\hat{\mathbf{x}}_{k+1}^{-}=\mathbf{f}\left(\hat{\mathbf{x}}_{k}^{+}, \mathbf{u}_{k}\right)
$$

2. The a priori error covariance in the $(k+1)$ th interval of time was obtained through:

$$
\mathbf{P}_{k+1}{ }^{-}=\mathbf{A P}_{k}{ }^{+} \mathbf{A}^{T}+\mathbf{W} \mathbf{Q} \mathbf{W}^{T} \text {; }
$$

3. The Kalman gain $\mathbf{K}_{k+1}$ was calculated through:

$$
\mathbf{K}_{k+1}=\mathbf{P}_{k+1}^{-} \mathbf{H}^{T}\left[\mathbf{H} \mathbf{P}_{k+1}^{-} \mathbf{H}^{T}+\mathbf{V R V}^{T}\right]^{-1} ;
$$

4. The a posteriori state estimate was calculated through:

$$
\hat{\mathbf{x}}_{k+1}^{+}=\hat{\mathbf{x}}_{k+1}^{-}+\mathbf{K}_{k+1}\left(\mathbf{z}_{k+1}-\mathbf{g}\left(\hat{\mathbf{x}}_{k+1}^{-}\right)\right) ;
$$

5. Finally, the a posteriori error covariance was obtained through:

$$
\mathbf{P}_{k+1}{ }^{+}=\left[\mathbf{I}-\mathbf{K}_{k+1} \mathbf{H}\right] \mathbf{P}_{k+1}^{-} .
$$

In the first case, where heterogeneity was not considered, density in $[\mathrm{veh} / \mathrm{km}]$ and average space-mean speed in $[\mathrm{km} / \mathrm{hr}]$ were considered as the state variables to characterize the system. The measured values of average space-mean speed values were taken as the measurement and the rate at which vehicles enter into the section from upstream and through the side road in [veh/hr] were provided as the inputs to the estimation scheme. Thus in this case, the parameters $\mathbf{x}, \mathbf{u}, \mathbf{z}$ and $\mathbf{H}$ were obtained as:

$$
\begin{aligned}
& \mathbf{x}(k)=\left[\begin{array}{c}
\rho(k) \\
v(k)
\end{array}\right] ; \\
& \mathbf{u}=\left[\begin{array}{c}
q_{\text {en }}(k) \\
q_{\text {side }}(k)
\end{array}\right] ; \\
& z(k)=v(k) ; \\
& \mathbf{H}=\left[\begin{array}{ll}
0 & 1
\end{array}\right] .
\end{aligned}
$$

Similarly, for the cases where all variables were considered in static PCU and dynamic TWU, the only change from the above was that the variables such as density and flow were considered in static PCU units and dynamic TWU units respectively.

Finally for the cases where different classes of vehicles were considered explicitly, these parameters were obtained as:

$$
\mathbf{x}(k)=\left[\begin{array}{c}
\rho_{1}(k) \\
\rho_{2}(k) \\
\rho_{3}(k) \\
v_{1}(k) \\
v_{2}(k) \\
v_{3}(k)
\end{array}\right] ;
$$

$$
\begin{aligned}
& \mathbf{u}(k)=\left[\begin{array}{c}
q_{e n}^{1}(k) \\
q_{e n}^{2}(k) \\
q_{e n}^{3}(k) \\
q_{\text {side }}^{1}(k) \\
q_{\text {side }}^{2}(k) \\
q_{\text {side }}^{3}(k)
\end{array}\right] ; \\
& \mathbf{z}(k)=\left[\begin{array}{c}
v_{1}(k) \\
v_{2}(k) \\
v_{3}(k)
\end{array}\right] ; \\
& \mathbf{H}=\left[\begin{array}{llllll}
0 & 0 & 0 & 1 & 0 & 0 \\
0 & 0 & 0 & 0 & 1 & 0 \\
0 & 0 & 0 & 0 & 0 & 1
\end{array}\right] .
\end{aligned}
$$

The other parameters such as $\mathbf{A}, \mathbf{W}$ and $\mathbf{V}$ for all the cases were derived from the corresponding model equations. The initial values of the state variables were assumed in all the cases and the above estimation schemes were implemented and corroborated using data collected from a road stretch in Chennai, India, as detailed in the following sections.

\section{Data Collection}

Data collection for the implementation and the corroboration of the estimation schemes presented in previous section were carried out using the video recording technique from the three sections $\mathrm{AB}, \mathrm{BC}$ and $\mathrm{AC}$ of the selected road in Chennai, India, as shown in Fig. 3. The section $\mathrm{BC}$ had one side entry in the middle and that is also included in this study. The traffic variables required for the implementation of the proposed scheme include the flow through the entry points and the side road and the average space mean speed of vehicles inside the study stretches. The variable that needs to be collected for corroboration of the scheme is traffic density.

Video data were collected at the entry and exit points of the selected sections of roadway and corresponding data from the side road were collected manually. The details of the data collection - the dates of data collection, the study stretch chosen, the duration of data collection and the traffic condition are enumerated in Table 3.

Data extraction was carried out manually due to the lack of any automated data extraction methods. Video data collected at the entry and exit points were analyzed in the laboratory to manually extract the required flow

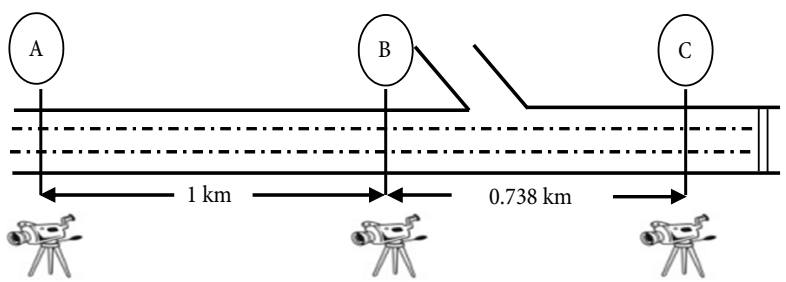

Fig. 3. Schematic sketch of study stretch showing the study sections 
Table 3. Details of data collection for implementation and corroboration of estimation schemes

\begin{tabular}{|c|c|c|c|c|}
\hline Section & Day & Date & $\begin{array}{l}\text { Duration } \\
\text { [minutes] }\end{array}$ & $\begin{array}{c}\text { Peak/ } \\
\text { Off } \\
\text { peak }\end{array}$ \\
\hline \multirow{3}{*}{$\begin{array}{l}\mathrm{AB} \\
(L=1 \mathrm{~km})\end{array}$} & 1 & 28 July 2009 & 61 & Peak \\
\hline & 2 & 30 June 2010 & 160 & $\begin{array}{c}\text { Off } \\
\text { peak }\end{array}$ \\
\hline & 3 & 03 December 2010 & 120 & $\begin{array}{c}\text { Off } \\
\text { peak }\end{array}$ \\
\hline \multirow{4}{*}{$\begin{array}{l}\mathrm{BC} \\
(L=0.738 \mathrm{~km})\end{array}$} & 4 & 29 December 2008 & 53 & Peak \\
\hline & 5 & 02 January 2009 & 54 & Peak \\
\hline & 6 & 12 September 2009 & 56 & Peak \\
\hline & 7 & 03 December 2010 & 117 & $\begin{array}{c}\text { Off } \\
\text { peak }\end{array}$ \\
\hline \multirow{2}{*}{$\begin{array}{l}\mathrm{AC} \\
(L=1.738 \mathrm{~km})\end{array}$} & 8 & 23 September 2010 & 124 & $\begin{array}{l}\text { Off } \\
\text { peak }\end{array}$ \\
\hline & 9 & 03 December 2010 & 117 & $\begin{array}{c}\text { Off } \\
\text { peak }\end{array}$ \\
\hline
\end{tabular}

data at a classified level and at every one-minute interval. The flow data at the entry and exit sections were extracted for every one-minute interval by classifying and counting the number of vehicles traveling in all three lanes. The spot speeds of vehicles of different classes passing the entry and exit sections were determined for every one minute by measuring the time taken to cross a known distance. The space-mean speeds were computed by taking the harmonic mean of spot speeds (May 1989). The average space-mean speed values of vehicles were computed by averaging the values at the entry and exit sections. The actual densities at every one-minute interval required for corroboration of the estimation scheme were determined using input-output analysis (May 1989). An aerial picture of the study sections at the start of video recording was taken to get a measure of the initial number of vehicles inside the sections required for the input output analysis. This was carried out by taking snapshots from either end of the study stretch. The photographs were overlapped and the number of vehicles inside the section was counted manually. The data extracted at classified levels were aggregated using static values of PCU and dynamic values of TWU to be used in the estimation schemes at aggregate levels. Using the collected data and assuming the initial values of the state variables, the proposed estimation scheme without incorporating heterogeneity and schemes with heterogeneity incorporated in different ways were implemented and corroborated as discussed in the following section.

\section{Evaluation of Incorporating Heterogeneity}

The results obtained using the above estimation schemes with and without heterogeneity incorporated were corroborated using the corresponding actual density values measured from field using the input output analysis. The performance of all these estimation schemes were quantified by calculating the Mean Absolute Percentage Error (MAPE) given by:

$$
M A P E=\left[\frac{1}{N} \cdot \sum_{k=1}^{N} \frac{\left|x_{e s t}(k)-x_{o b s}(k)\right|}{x_{o b s}(k)}\right] \cdot 100 \%,
$$

where: $x_{\text {est }}(k), x_{o b s}(k)$ are the estimated and observed or measured values of the state variable during the $k$ th interval of time respectively and $N$ is the total number of observations. The MAPE values for traffic density using all the proposed schemes are given in Table 4 and Fig. 4 shows a comparison of these results.

It can be seen that use of dynamic TWU does not improve the results for most days as compared to use of static PCU values. At the classified level, the scheme considering interaction where speed of a certain class of vehicle in a heterogeneous mix was assumed to be influenced by the presence of all other vehicles in the roadway, is reasonable. When heterogeneity was incorporated at aggregate and at classified levels, inclusion of different classes of vehicles into the model did not significantly improve the performance compared to that

Table 4. MAPE for density estimation

\begin{tabular}{|c|c|c|c|c|c|c|}
\hline \multirow{3}{*}{ Section } & \multirow{3}{*}{ Day } & \multicolumn{5}{|c|}{ MAPE [\%] } \\
\hline & & \multirow{2}{*}{$\begin{array}{c}\text { Without incorporating } \\
\text { heterogeneity }\end{array}$} & \multicolumn{4}{|c|}{ Heterogeneity incorporated } \\
\hline & & & Static PCU & $\begin{array}{l}\text { Dynamic } \\
\text { TWU }\end{array}$ & $\begin{array}{l}\text { Multi-class without } \\
\text { interaction }\end{array}$ & $\begin{array}{l}\text { Multi-class with } \\
\text { interaction }\end{array}$ \\
\hline \multirow{3}{*}{$\begin{array}{l}\mathrm{AB} \\
(L=1 \mathrm{~km})\end{array}$} & 1 & 20.8 & 20.6 & 19.9 & 22.8 & 23.6 \\
\hline & 2 & 8.0 & 9.2 & 11.3 & 10.4 & 9.7 \\
\hline & 3 & 11.1 & 11.8 & 13.6 & 10.0 & 9.8 \\
\hline \multirow{4}{*}{$\begin{array}{l}\mathrm{BC} \\
(L=0.738 \mathrm{~km})\end{array}$} & 4 & 13.9 & 13.9 & 23.3 & 16.4 & 15.7 \\
\hline & 5 & 11.3 & 15.8 & 22.1 & 14.7 & 14.5 \\
\hline & 6 & 23.8 & 24.5 & 29.3 & 23.7 & 23.8 \\
\hline & 7 & 17.1 & 18.2 & 25.6 & 17.4 & 17.7 \\
\hline \multirow{2}{*}{$\begin{array}{l}\mathrm{AC} \\
(L=1.738 \mathrm{~km})\end{array}$} & 8 & 16.3 & 13.6 & 25.9 & 15.8 & 14.6 \\
\hline & 9 & 13.1 & 9.2 & 13.6 & 10.7 & 10.1 \\
\hline
\end{tabular}




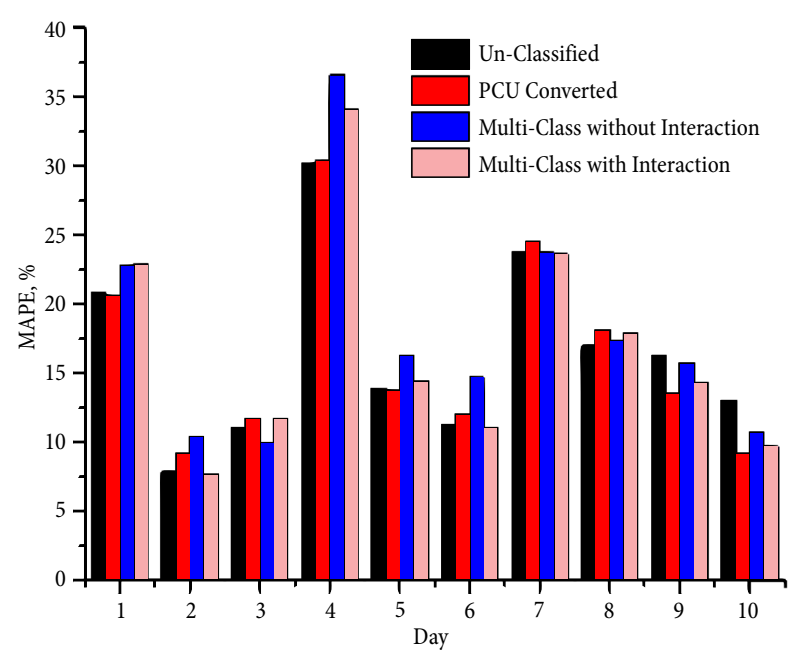

Fig. 4. Comparison of performance of density estimation schemes

aggregate level using static PCU. Finally, the comparison of schemes where heterogeneity was not explicitly considered and where heterogeneity was considered in terms of static PCU, shows that the results were comparable and the inclusion of heterogeneity is not making any significant difference in estimating traffic density at the aggregate level.

\section{Summary and Conclusions}

Traffic flow conditions prevailing in India and many other developing countries are highly complex with its heterogeneity and lack of lane discipline making analysis and modeling of traffic difficult. It is in general expected that in order to characterize the heterogeneous traffic more accurately, the heterogeneity needs to be incorporated into the models irrespective of whether one is dealing with microscopic, macroscopic or mesoscopic modeling.

The present study reported a systematic comparison of the effect of including heterogeneity in a macroscopic modeling taking real time congestion analysis as an example. A non-continuum macroscopic model was proposed in this study for congestion analysis on Indian roads. Traffic density was used to indicate traffic congestion and was estimated using a model-based estimation scheme developed using the extended Kalman filter. Heterogeneity was incorporated at aggregate level and at classified levels. Both static values of PCU and dynamic values of TWU were used to aggregate the traffic. At classified level, the heterogeneous traffic was classified into different vehicle groups and explicitly included into the model. The proposed schemes with and without heterogeneity were corroborated using data measured from a road stretch in Chennai, India. The results showed that the incorporation of heterogeneity did not have a significant effect in estimating traffic density at the macroscopic level.

It is generally believed that the effects of heterogeneity need to be incorporated explicitly in modelling heterogeneous traffic. But the present study illustrates that this need not be the case for all models of macroscopic nature involving heterogeneous traffic. This would results in a reduced number of equations and associated parameters, which will lead to reduced calibration, computational and data requirements with respect to field implementation. In future, this approach can be used for developing congestion prediction and travel time prediction applications for heterogeneous traffic suitable for real time field implementation.

\section{Acknowledgements}

The authors acknowledge the support provided by the Ministry of Urban Development, Government of India through grant No. N-11025/30/2008-UCD and the Indo-US Science and Technology Forum through grant No. IUSSTF/JC-Intelligent Transportation Systems/95-2010/2011-12.

\section{References}

Anand, R. A.; Vanajakshi, L.; Subramanian, S. C. 2011. Traffic density estimation under heterogeneous traffic conditions using data fusion, in Proceedings of the 2011 IEEE Intelligent Vehicles Symposium (IV), 5-9 June, 2011, BadenBaden, Germany, 31-36. http://dx.doi.org/10.1109/IVS.2011.5940397

Arasan, V. T.; Krishnamurthy, K. 2008. Effect of traffic volume on PCU of vehicles under heterogeneous traffic conditions, Road \& Transport Research 17(1): 32-49.

Chandra, S.; Kumar, V.; Sikdar, P. K. 1995. Dynamic PCU and estimation of capacity of urban roads, Indian Highways 23(4): 17-28.

Chandra, S.; Kumar, U. 2003. Effect of lane width on capacity under mixed traffic conditions in India, Journal of Transportation Engineering 129(2): 155-160.

http://dx.doi.org/10.1061/(ASCE)0733-947X(2003)129:2(155)

Chen, L.; May, A. D. 1987. Traffic detector errors and diagnostics, Transportation Research Record 1132: 82-93.

Coddington, E. A. 1989. An Introduction to Ordinary Differential Equations. Dover Publications. 320 p.

Gazis, D. C.; Szeto, M. W. 1974. Design of density-measuring systems for roadways, Transportation Research Record 495: 44-52.

Hall, F. L. 2001. Traffic Stream Characteristics, in Gartner, N.; Messer, C. J.; Rathi, A. K. (Eds.). Traffic Flow Theory: A State-of-the-Art Report, 2-1-2-37.

Hoogendoorn, S. P.; Bovy, P. H. L. 2001. State-of-the-art of vehicular traffic flow modelling, Proceedings of the Institution of Mechanical Engineers, Part I: Journal of Systems and Control Engineering 215(4): 283-303. http://dx.doi.org/10.1177/095965180121500402

Ioannou, P. A.; Chien, C. C. 1993. Autonomous intelligent cruise control, IEEE Transactions on Vehicular Technology 42(4): 657-672. http://dx.doi.org/10.1109/25.260745

IRC. 1990. Guidelines for Capacity of Urban Roads in Plain Areas. Indian Roads Congress (IRC-106), New Delhi, India. $12 \mathrm{p}$.

Kalman, R. E. 1960. A new approach to linear filtering and prediction problems, Transactions of the ASME-Journal of Basic Engineering (Series D) 82: 35-45. 
Logghe, S.; Immers, B. 2003. Heterogeneous traffic flow modelling with the LWR model using passenger-car equivalents, in Proceedings of the 10th World Congress on ITS, Madrid, Spain. 15 p. (CD).

May, A. D. 1989. Traffic Flow Fundamentals. 1st edition. Prentice Hall. 464 p.

Maybeck, P. S. 1979. Stochastic Models, Estimation and Control. Vol. 1. Academic Press. 444 p.

Maybeck, P. S. 1982a. Stochastic Models, Estimation and Control. Vol. 2. Academic Press. 290 p.

Maybeck, P. S. 1982b. Stochastic Models, Estimation and Control. Vol. 3. Academic Press. 291 p.

Nahi, N. E.; Trivedi, A. N. 1973. Recursive estimation of traffic variables: section density and average speed, Transportation Science 7(3): 269-286.

http://dx.doi.org/10.1287/trsc.7.3.269

Nanthawichit, C.; Nakatsuji, T.; Suzuki, H. 2003. Application of probe-vehicle data for real-time traffic-state estimation and short-term travel-time prediction on a freeway, Transportation Research Record 1855: 49-59. http://dx.doi.org/10.3141/1855-06

Okutani, I.; Stephanedes, Y. J. 1984. Dynamic prediction of traffic volume through Kalman filtering theory, Transportation Research Part B: Methodological 18(1): 1-11. http://dx.doi.org/10.1016/0191-2615(84)90002-X

Padiath, A.; Vanajakshi, L.; Subramanian, S. C.; Manda, H. 2009. Prediction of traffic density for congestion analysis under Indian traffic conditions, in Proceedings of the 12th International IEEE Conference on Intelligent Transportation Systems, 2009. ITSC'09, 4-7 October, 2009, St. Louis, Missouri, USA, 1-6. http://dx.doi.org/10.1109/ITSC.2009.5309716

Padiath, A. S.; Vanajakshi, L. D.; Subramanian, S. C. 2010. Estimation of Traffic Density under Indian Traffic Conditions, in TRB 89th Annual Meeting Compendium of Papers DVD, 10-14 January, 2010, Washington, DC, USA. 15 p. (DVD).

Péter, T. 2012. Modeling nonlinear road traffic networks for junction control, International Journal of Applied Mathematics and Computer Science 22(3): 723-732.

Swaroop, D.; Hedrick, J. K.; Chien, C. C.; Ioannou, P. 1994. A comparision of spacing and headway control laws for automatically controlled vehicles, Vehicle System Dynamics 23(1): 597-625. http://dx.doi.org/10.1080/00423119408969077

Tang, T. Q.; Huang, H. J.; Zhao, S. G.; Shang, H. Y. 2009. A new dynamic model for heterogeneous traffic flow, Physics Letters A 373(29): 2461-2466.

http://dx.doi.org/10.1016/j.physleta.2009.05.006

Thankappan, A.; Vanajakshi, L. D. 2012. Development of optimized traffic stream models under heterogeneous traffic conditions, in TRB 91st Annual Meeting Compendium of Papers DVD, 22-26 January, 2012, Washington, DC, USA. 18 p. (DVD).

Turner, S.; Albert, L.; Gajewski, B.; Eisele, W. 2000. Archived intelligent transportation system data quality: preliminary analyses of San Antonio TransGuide data, Transportation Research Record 1719: 77-84. http://dx.doi.org/10.3141/1719-10

Tyagi, V.; Darbha, S.; Rajagopal, K. R. 2009. A review of the mathematical models for traffic flow, International Journal of Advances in Engineering Sciences and Applied Mathematics 1(1): 53-68.

http://dx.doi.org/10.1007/s12572-009-0005-8
Vanajakshi, L.; Rilett, L. R. 2004. Loop detector data diagnostics based on conservation-of-vehicles principle, Transportation Research Record 1870: 162-169.

http://dx.doi.org/10.3141/1870-21

Wang, Y.; Papageorgiou, M. 2005. Real-time freeway traffic state estimation based on extended Kalman filter: a general approach, Transportation Research Part B: Methodological 39(2): 141-167. http://dx.doi.org/10.1016/j.trb.2004.03.003

Wong, G. C. K.; Wong, S. C. 2002. A multi-class traffic flow model - an extension of LWR model with heterogeneous drivers, Transportation Research Part A: Policy and Practice 36(9): 827-841.

http://dx.doi.org/10.1016/S0965-8564(01)00042-8 